\title{
The value of cytoplasmic Y-box-binding protein 1 as a prognostic marker for breast cancer in Korean
}

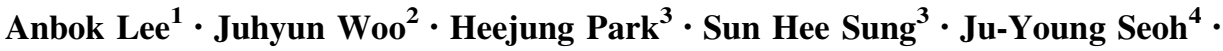 \\ Woosung Lim² ${ }^{2}$ Byung-In Moon ${ }^{2}$
}

Received: 6 January 2015 / Accepted: 7 July 2015/Published online: 21 July 2015

(c) The Author(s) 2015. This article is published with open access at Springerlink.com

\begin{abstract}
Background The human Y-box-binding protein 1 (YB-1) is a member of the DNA/RNA-binding family of proteins that regulates transcription and translation of genes. Previous studies suggest that YB-1 may have an oncogenic role in various cancers. In this study, we evaluate the prognostic value of cytoplasmic $\mathrm{YB}-1$ with respect to breast cancer.

Methods Immunohistochemical staining study was performed with YB-1 using tissue block from 233 patients with invasive ductal carcinoma. Patients were divided into two groups according to expression of cytoplasmic YB-1 in tumor cell (high versus low). The relationship between the expression of YB-1, clinicopathological characteristics and breast cancer prognosis was analyzed.

Results Hormone receptor negativity, worse histologic and nuclear grade, high tumor stage, lymphovascular invasion and high Ki67 $(\geq 14 \%)$ were related with the
\end{abstract}

B.-I. Moon and W. Lim contributed equally to the research of the paper.

Woosung Lim

limw@ewha.ac.kr

$\bowtie$ Byung-In Moon

mbit@ewha.ac.kr

1 Department of Surgery, Busan Paik Hospital, Inje University, College of Medicine, Busan, Korea

2 Department of Surgery, School of Medicine, Mokdong Hospital, Ewha Womans University, Seoul, Korea

3 Department of Pathology, School of Medicine, Mokdong Hospital, Ewha Womans University, Seoul, Korea

4 Department of Microbiology, School of Medicine, Ewha Womans University, Seoul, Korea increased expression of cytoplasmic YB-1 in tumor cell $(p<0.05)$. Although there was no significant difference in relapse-free survival (RFS) between the two groups $(p=0.412)$, difference in overall survival (OS) was statistically significant $(p=0.035)$. In multivariate analysis for OS, YB-1 was an independent prognostic factor $(p=0.043)$.

Conclusion This suggests that the increased expression of cytoplasmic YB-1 in tumor cells can be regarded as an independent prognostic factor for breast cancer, related to poor prognostics. Expression of cytoplasmic YB-1 in cancer cell could be used as an independent prognostic marker for predicting OS in breast cancer.

Keywords YBX1 protein · Human · Cytoplasm ·

Prognosis · Breast neoplasm

\section{Introduction}

Breast cancer is the most common cancer for women and its incidence is gradually increasing [1]. It is a kind of heterogeneous disease due to its broad spectrum of morphological features, clinical manifestations, and treatment responses [2]. Therefore, it needs various makers for predicting prognosis and many previous studies have searched for new markers for prognosis and treatment in breast cancer.

Recently, the human Y-box-binding protein 1 (YB-1) has been suggested as a new prognostic marker in many studies. YB-1 is a nucleic acid binding protein, which is located in chromosome 1p34. The process that cold shock domain of YB-1 binds with the Y-box in the gene promoter regulates gene transcription and translation. YB-1 is also 
known to be involved in DNA repair, splicing, stabilization, cell proliferation and regeneration [3-5].

Several studies have reported on the oncogenic role of YB-1 and mainly focused on the nuclear expression of YB-1 in tumor cell. Nuclear expression of YB-1 in tumor cells is known to be associated with poor prognosis in various types of cancer [6-9]. It has been also reported to be involved in multidrug resistance. Translocation of YB1 into nucleus activates the transcription of multidrug resistance (MDR) 1 gene and the expression of P-glycoprotein, subsequently producing drug resistance [10]. In matter of breast cancer, YB-1 has been reported to induce human epidermal growth factor receptor (HER)-2. The nuclear expression of YB-1 has been introduced to be related with the negative expression of estrogen receptor (ER) and progesterone receptor (PR) [11]. Furthermore, according to previous report, it has been also known that cytoplasmic expression of YB-1 is related with cancer prognosis [12].

Based on these considerations, this study aims to evaluate the significance of cytoplasmic YB-1 expression for prognosis prediction in breast cancer.

\section{Methods}

\section{Patient selection}

Between January 2003 and December 2008, a total of 538 patients underwent breast cancer surgery at the Department of Surgery, Ewha Womans University Mokdong Hospital. Among these patients, 233 patients were finally enrolled in this study. Patient exclusion criteria were as follows: (1) male, (2) patient who received neoadjuvant chemotherapy, (3) ductal carcinoma in situ, (4) distant metastasis at the time of diagnosis, (5) $2+$ for HER2 by immunohistochemistry (IHC), (6) death unrelated with breast cancer, (7) patient with other concomitant cancer (except papillary thyroid cancer, cervical intraepithelial neoplasia:CIN, nonmelanoma skin cancer) and (8) patient with no paraffin block or inappropriate paraffin block for study. Molecular subtype was defined as follows: luminal A (ER+ and/or $\mathrm{PR}+, \mathrm{Ki} 67<20$ and HER2-), luminal B (ER+ and/or $\mathrm{PR}+, \mathrm{Ki} 67 \geq 20$ and/or HER2+), HER2+ enrich (ER-, PR - and HER2+) and triple negative breast cancer (TNBC) (ER-, PR - and HER2-). Cases with 2+ score were excluded from this study because fluorescent in situ hybridization (FISH) was not performed at our institute prior to 2006.

This study was approved by the Institutional Review Board of Ewha Womans University Mokdong Hospital (IRB approval number, 12-16A-18).

\section{Immunohistochemical staining}

Formalin-fixed paraffin sections $(2.5 \mu \mathrm{m})$ of tumor tissues were deparaffinized with xylene and rehydrated in graded alcohols. Immunohistochemical staining was done with the Dako Envision system, which uses dextran polymers conjugated with horseradish peroxidase (Dako, Carpinteria, CA, USA). For antigen retrieval, the slides in a target retrieval solution ( $\mathrm{pH}$ 6.0; DAKO) were boiled for $10 \mathrm{~min}$ in a microwave oven. Tissue sections were incubated in a humidified box at $4{ }^{\circ} \mathrm{C}$ overnight with a primary antibody (Ab) against YB-1 (1:100 dilutions, Novus USA). After incubation, the sections were treated with an Envision+System-HRP secondary Ab (DaKo). Color development was conducted using 3-amino-9-ethylcarbazole (AEC) (DAKO) as a chromogen. Finally, all sections were counterstained with hematoxylin (DaKo). Negative control sections were treated in the same manner except if they were incubated with Tris-buffered saline without primary $\mathrm{Ab}$.

\section{Interpretation}

To evaluate the expression for YB-1, two pathologists independently reviewed the slides using an immunoreactivity score (IRS) without any patient information. IRS was evaluated by multiplying the intensity score by percentage of positive tumor cells. The staining results were scored by (1) intensity scores: 0 (no staining), 1 (weak), 2 (moderate), 3(strong); and (2) percentage of positive tumor cells: 0 $(0-10 \%), 1$ (11-25\%), 2 (26-50\%), 3 (51-75\%), 4 (76-100\%). Patients were divided into two groups according to IRS. Scores $0-4$ were considered as the low expression group (low group) and 5-12 were considered as the high expression group (high group) (Fig. 1). We determined score which showed best statistical results for prognostic factors and prognosis as cutoff value. Expressions of Hormone receptor and HER2+ were determined based on IHC profiles. ER/PR positivity was defined when more than $10 \%$ of cancer cells were stained with more than $1+$ of intensity. In respect to c-erbB2 expression, score of $3+$ was regarded as positive and scores of $0,1+$ were regarded as negative.

\section{Statistical analysis}

Pearson's Chi-square test and Fisher's exact test were used to compare patient's clinicopathologic characteristics. Relapse-free survival (RFS) was defined as a length of time from surgery to relapse of cancer and overall survival (OS) was defined as from surgery to breast cancer related death 

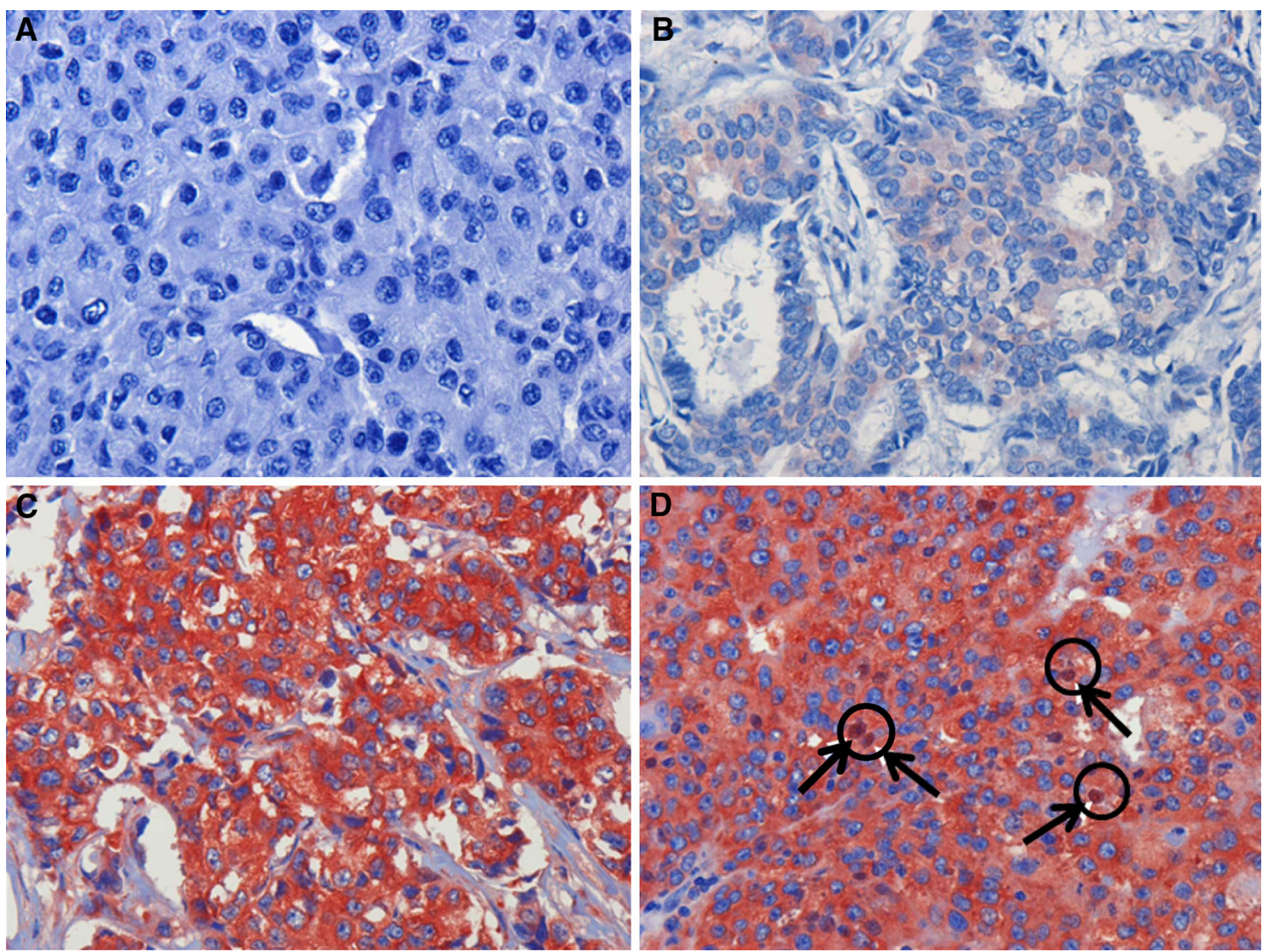

Fig. 1 Four different patterns of microscopic findings of immunohistochemical staining for YB-1 expression in showing this study. a YB-1 was not expressed in cytoplasm and nucleus. b YB-1 was

and analyzed by the Kaplan-Meier method. Univariate analysis was performed using Log-rank test and multivariate analysis was performed using Cox's proportional hazard model with $95 \%$ confidence interval (CI). The mean value comparison of chemotherapeutic agents was conducted by the Mann-Whitney test. All statistical tests were performed by SPSS (version 18, SPSS Inc., Chicago, IL, USA) and $p$ value $<0.05$ was considered as statistically significant in all results.

\section{Results}

\section{Clinicopathologic characteristics of all patients}

A total of 233 females were included in this study and their mean age was $50.2 \pm 11.0$ years. Median follow-up duration was $59.0 \pm 25.1$ months. Among the 233 patients, 14 patients $(6.0 \%)$ were younger than 35 years old. 106 patients $(45.5 \%)$ received breast conserving surgery (BCS) and 127 patients $(54.5 \%)$ underwent mastectomy. expressed weakly in cytoplasm. c YB-1 was expressed strongly in cytoplasm but not in the nucleus. d YB-1 was expressed strongly in both cytoplasm and nucleus (black arrows and circles)

In terms of histologic types, there were 227 cases (97.4\%) of invasive ductal carcinoma and 6 cases (2.6\%) of invasive lobular carcinoma (ILC). Table 1 shows the clinicopathologic characteristics of all patients.

\section{Expression of YB-1 in breast cancer cell and relationship between expression of cytoplasmic YB-1 and prognostic factors}

Cytoplasmic YB-1 expression in cancer cell was evaluated using IRS. High expressions of YB-1 (score 5-12) were observed in $112(48.1 \%)$ patients and low expressions (score 0-4) were observed in 121 patients (Fig. 1).

Table 2 presents the relationship between expression of cytoplasmic YB-1 and prognostic factors. High expression of cytoplasmic YB-1 was more frequently observed in ER, PR negative tumor than in positive tumor $(p<0.001)$. Regarding to molecular subtypes, there were more HER2 type tumor and TNBC in the high expression group $(p<0.001)$. High expression of cytoplasmic YB-1 was related with histologic and nuclear grade III tumors $(p<0.001)$. Tumors with high ki-67 were more frequently 
Table 1 Clinicopathologic characteristics in this study

\begin{tabular}{|c|c|}
\hline Patient characteristics & Number of patients (\%) \\
\hline \multicolumn{2}{|l|}{ Age } \\
\hline$<35$ & $14(6.0 \%)$ \\
\hline$\geq 35$ & $219(94.0 \%)$ \\
\hline \multicolumn{2}{|l|}{ Operation } \\
\hline BCS & $106(45.5 \%)$ \\
\hline MRM & $127(54.5 \%)$ \\
\hline \multicolumn{2}{|l|}{ Histology } \\
\hline IDC & $227(94.4 \%)$ \\
\hline ILC & $6(5.6 \%)$ \\
\hline \multicolumn{2}{|l|}{ ER } \\
\hline Negative & $73(31.3 \%)$ \\
\hline Positive & $160(68.7 \%)$ \\
\hline \multicolumn{2}{|l|}{ PR } \\
\hline Negative & $85(36.5 \%)$ \\
\hline Positive & $148(63.5 \%)$ \\
\hline \multicolumn{2}{|l|}{ c-erbB2 } \\
\hline Negative & $156(67.0 \%)$ \\
\hline Positive & $77(33.3 \%)$ \\
\hline \multicolumn{2}{|l|}{ Histologic grade } \\
\hline 1 & $72(30.9 \%)$ \\
\hline 2 & $101(43.3 \%)$ \\
\hline 3 & $55(23.6 \%)$ \\
\hline Unknown & $5(2.2 \%)$ \\
\hline \multicolumn{2}{|l|}{ Nuclear grade } \\
\hline 1 & $39(16.7 \%)$ \\
\hline 2 & $130(55.8 \%)$ \\
\hline 3 & $59(25.3 \%)$ \\
\hline Unknown & $5(2.2 \%)$ \\
\hline \multicolumn{2}{|l|}{ Ki67 } \\
\hline$<20$ & $136(58.4 \%)$ \\
\hline$\geq 20$ & $97(41.6 \%)$ \\
\hline \multicolumn{2}{|l|}{$T$ stage } \\
\hline 1 & $116(49.8 \%)$ \\
\hline 2 & $103(44.2 \%)$ \\
\hline 3 & $13(5.6 \%)$ \\
\hline 4 & $1(0.4 \%)$ \\
\hline \multicolumn{2}{|l|}{$N$ stage } \\
\hline 0 & $145(62.8 \%)$ \\
\hline 1 & $55(23.8 \%)$ \\
\hline 2 & $18(7.8 \%)$ \\
\hline 3 & $13(5.6 \%)$ \\
\hline
\end{tabular}

$B C S$ breast conserving surgery, $M R M$ modified radical mastectomy, $I D C$ invasive ductal carcinoma, $I L C$ invasive lobular carcinoma, $E R$ estrogen receptor, $P R$ progesterone receptor

observed in high expression group $(p<0.001)$. Tumor stage was also significantly related with high cytoplasmic YB-1 expression $(p=0.017)$.
Table 2 Comparisons of clinicopathologic results according to expression of YB-1

\begin{tabular}{|c|c|c|c|}
\hline \multirow[t]{2}{*}{ Patient characteristics } & \multicolumn{2}{|c|}{ Number of patients (\%) } & \multirow[t]{2}{*}{$p$ value } \\
\hline & Low $(n=121)$ & $\operatorname{High}(n=112)$ & \\
\hline Age & & & 0.210 \\
\hline$<35$ & $5(4.1 \%)$ & $9(8.0 \%)$ & \\
\hline$\geq 35$ & $116(95.9 \%)$ & $103(92.0 \%)$ & \\
\hline Operation & & & 0.117 \\
\hline BCS & $61(50.4 \%)$ & $45(40.2 \%)$ & \\
\hline MRM & $60(49.6 \%)$ & $67(59.8 \%)$ & \\
\hline Histology & & & 0.119 \\
\hline IDC & $116(95.9 \%)$ & $111(99.1 \%)$ & \\
\hline ILC & $5(4.1 \%)$ & $1(0.9 \%)$ & \\
\hline ER & & & $<0.001$ \\
\hline Negative & $18(14.9 \%$ & $55(49.1 \%)$ & \\
\hline Positive & $103(85.1 \%)$ & $57(50.9 \%)$ & \\
\hline PR & & & $<0.001$ \\
\hline Negative & $28(23.1 \%)$ & $57(50.9 \%)$ & \\
\hline Positive & $93(76.9 \%)$ & $55(49.1 \%)$ & \\
\hline c-erbB2 & & & 0.051 \\
\hline Negative & $88(72.7 \%)$ & $68(60.7 \%)$ & \\
\hline Positive & $33(27.3 \%)$ & $44(39.3 \%)$ & \\
\hline Molecular subtype & & & $<0.001$ \\
\hline Luminal A & $64(52.9 \%)$ & $30(26.8 \%)$ & \\
\hline Luminal B & $41(33.9 \%)$ & $32(28.6 \%)$ & \\
\hline HER2 enrich & $9(7.4 \%)$ & $25(22.3 \%)$ & \\
\hline TNBC & $7(5.8 \%)$ & $25(22.3 \%)$ & \\
\hline Histologic grade & & & $<0.001$ \\
\hline 1 & $52(44.4 \%)$ & $20(18.0 \%)$ & \\
\hline 2 & $52(44.4 \%)$ & $49(44.1 \%)$ & \\
\hline 3 & $13(11.1 \%)$ & $42(37.8 \%)$ & \\
\hline Unknown & 4 & 1 & \\
\hline Nuclear grade & & & $<0.001$ \\
\hline 1 & $30(25.6 \%)$ & $9(8.1 \%)$ & \\
\hline 2 & $72(61.5 \%)$ & $58(52.3 \%)$ & \\
\hline 3 & $15(12.8 \%)$ & $44(39.6 \%)$ & \\
\hline Unknown & 4 & 1 & \\
\hline Ki67 & & & $<0.001$ \\
\hline$<20$ & $86(71.1 \%)$ & $50(44.6 \%)$ & \\
\hline$\geq 20$ & $35(28.9 \%)$ & $62(55.4 \%)$ & \\
\hline$T$ stage & & & 0.017 \\
\hline 1 & $70(57.9 \%)$ & $46(41.1 \%)$ & \\
\hline 2 & $47(38.8 \%)$ & $56(50.0 \%)$ & \\
\hline 3 & $3(2.5 \%)$ & $10(8.9 \%)$ & \\
\hline 4 & $1(0.8 \%)$ & 0 & \\
\hline$N$ stage & & & 0.070 \\
\hline 0 & $78(64.5 \%)$ & $68(60.7 \%)$ & \\
\hline 1 & $33(27.3 \%)$ & $22(19.6 \%)$ & \\
\hline 2 & $6(5.0 \%)$ & $13(11.6 \%)$ & \\
\hline 3 & $4(3.3 \%)$ & $9(8.0 \%)$ & \\
\hline
\end{tabular}

$B C S$ breast conserving surgery, MRM modified radical mastectomy, $I D C$ invasive ductal carcinoma, ILC invasive lobular carcinoma, $E R$ estrogen receptor, $P R$ progesterone receptor, $T N B C$ triple negative breast cancer 


\section{Prognosis according to cytoplasmic YB-1 expression}

Kaplan-Meier analysis was conducted to evaluate RFS and OS according to expression of cytoplasmic YB-1. In RFS, there was no significant difference between the two groups ( $p=0.412$ ) (Fig. 2a). In terms of OS, 5-year survival rates of the high and low group were 92 and $98 \%$, respectively, showing a significant difference between the two groups $(p=0.035)$ (Fig. 2b). Multivariate analysis was performed using Cox proportionate hazard regression model and high expression of cytoplasmic YB-1 was confirmed as an independent prognostic factor for OS $(p=0.043)$ (Table 3).

\section{Discussion}

YB-1 is a DNA- and RNA-binding protein, which is located on chromosome 1p34 and involved in various cellular processes. This protein plays a role in DNA replication, repair, transcription, pre-mRNA splicing, and mRNA translation [3-5]. It was reported that the expression of YB-1 increases in cancer cells and its high expression or expression in nucleus were associated with poor prognosis in many types of cancer [6-9, 13]. Also, this nuclear expression of YB-1 in tumor cell activates gene transcription for protein and relates to multidrug resistance and ionizing radiation [10, 14].

In breast cancer, the oncogenic role of YB-1 has been widely studied. Previous studies showed that the localization of YB-1 in nucleus was associated with poor prognosis as well as with poor prognostic factors $[8,11,15]$. Some studies showed that the nuclear YB-1 expression was positively correlated with the HER2 expression and suggested the inhibition of YB-1 could be a good therapeutic target for HER2 type tumor [15-17]. Ito et al. [18] found that the alteration of YB-1 after neoadjuvant chemotherapy might modify the crosstalk between ER and HER2 pathways, leading to endocrine therapy resistance. According to the study of $\mathrm{Li}$ et al. [12], high expression of YB-1 in cytoplasm of cancer cell was significantly associated with poor prognosis in squamous esophageal cancer. However, their study did not show a significant relationship between YB-1 expression and prognostic factors.

In this study, we could observe high cytoplasmic expressions of YB-1 in 112 (48.1\%) patients and YB-1 was also expressed in cancer cell nucleus. Nuclear expression of YB-1 was also significantly related with poor prognostic factors (ER and PR negativity, high histologic and nuclear grade, high Ki-67 expression) and poor OS (data were not shown). However, such an expression was observed in 26 out of 233 cases $(11.2 \%)$ and this was a relatively small proportion compared to previous studies $[8,11,15]$. Some previous studies reported that nuclear localization of YB-1 was induced by growth factors and cytokines and related with tumor progression [19-21]. This means that more advanced and aggressive cancer expresses more amounts of nuclear YB-1. In our study, tumors were relatively in their early stages compared to those of previous studies. We consider this finding may have contributed to the lower nuclear expression of YB-1. Actually, among the 26 patients with nuclear expression of YB-1,
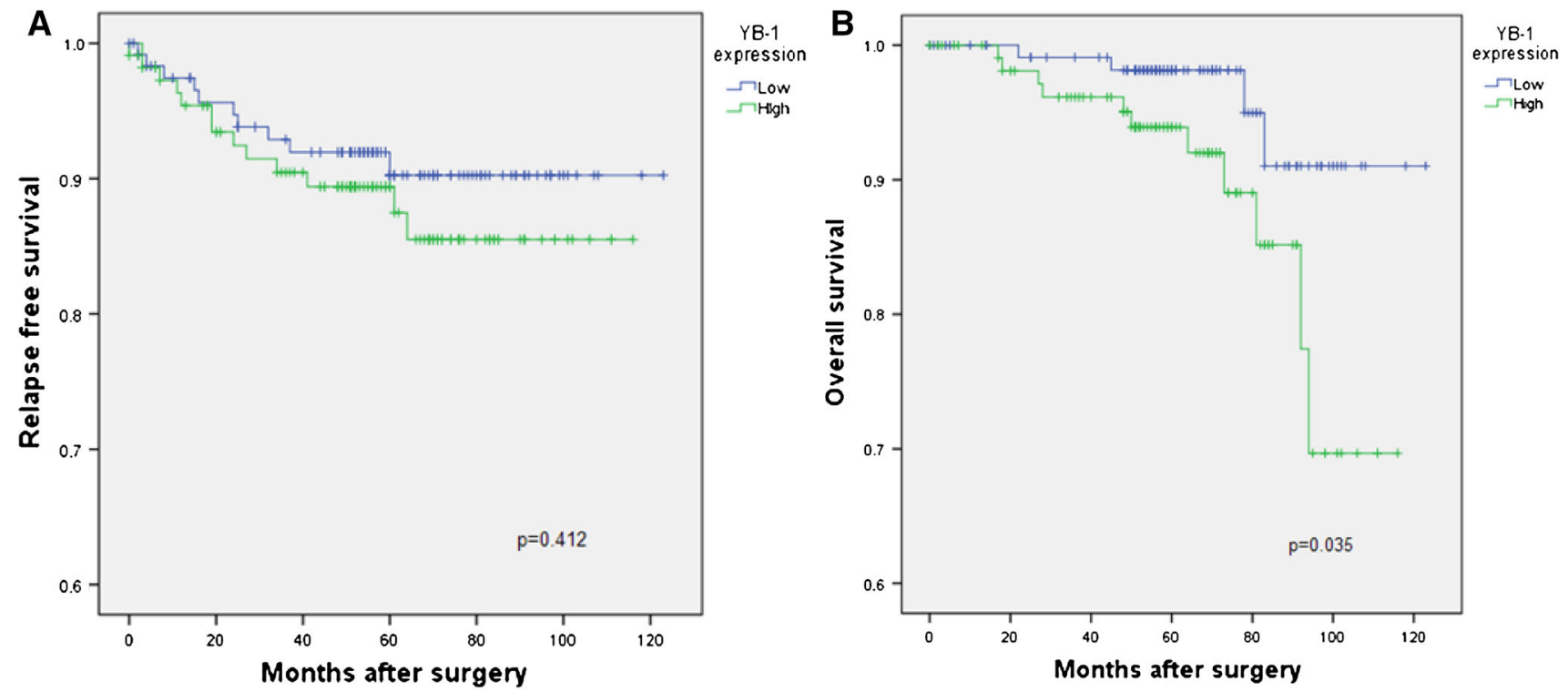

Fig. 2 Relapse-free survival and overall survival analysis according to YB-1 expression. a Relapse-free survival according to YB-1 expression, b overall survival analysis according to YB-1 expression 
Table 3 Multivariate analysis for overall survival

\begin{tabular}{llllll}
\hline & Wald statistic & Degree of freedom & $p$ value & Hazard ratio & CI (95 \%) \\
\hline Cytoplasmic YB-1 expression & 4.079 & 1 & 0.043 & 3.259 & $1.035-10.255$ \\
Nodal stage & 5.411 & 1 & 0.020 & 3.583 & $1.223-10.501$ \\
\hline
\end{tabular}

Including covariates are as follows: age $(<35, \geq 35)$, hormonal receptor status (ER/PR), c-erbB2 status, Tumor stage $(T 1+T 2 / T 3+T 4)$, nodal stage $(N 0 / N 1+N 2+N 3), \mathrm{Ki}-67(<20, \geq 20)$

both nucleus and cytoplasm of YB-1 were observed in 24 patients $(99.1 \%)$ and these tumors showed relatively high $T$ stage, high histologic and nuclear grade, more aggressive biologic features compared to the tumors expressed YB-1 only in cytoplasm.

Our study showed that high IRS group was significantly related with ER/PR positive, high Ki-67, high histologic grade, high nuclear grade and high $T$ stage $(p<0.05)$. Although, c-erbB2 was not significantly related with the high expression of YB-1, there was a tendency that tumor with positive c-erbB2 expressed high YB-1 $(p=0.051)$. The High group was also associated with poor OS $(p=0.035)$, but not with RFS $(p=0.412)$. Subsequently, recurrence patterns according to YB-1 expression were analyzed based on a total of 23 tumor recurrences that were observed. Among them, 13 recurrence cases were observed in the high group and the other 10 recurrence cases in the low group. There were a total of 17 systemic recurrences where $12(70.6 \%)$ were observed in the high group and 5 $(29.4 \%)$ in the low group $(p=0.022)$. These recurrence patterns might be contributing towards the significant difference in OS between the two groups (Table 4). According to previous studies, elevated expression of YB-1 in cancer cell promotes epithelial-mesenchymal transition in various cancers including breast cancer, and this is also related with angiogenesis in tumor microenvironment [2224]. We think that this mechanism might affect more frequent systemic recurrence and poor OS in the high group. Additionally, during the molecular subgroup analysis for recurrence cases in the high group, three $(23.1 \%)$ cases were TNBC, two $(15.4 \%)$ were HER2+ type tumors and the rest were luminal type (A or B) tumors. In the low group, there was no TNBC while there was one $(10.0 \%)$ and nine cases $(90.0 \%)$ of HER $2+$ type and luminal (A or B) types, respectively. Yi et al. [25] reported that TNBC patients had shorter OS than non-TNBC patients despite favorable response to palliative doxorubicin based chemotherapy. They assumed that this might be due to frequent resistance to chemotherapeutic agent in TNBC. Our prognostic results might be also affected by this mechanism.

There were some limitations of this study. Because our cases were composed of relatively early stage tumors compared to previous studies, there may be fewer cancer
Table 4 Recurrence patterns according to YB-1

\begin{tabular}{lccc}
\hline Recurrence pattern & Low group & High group & $p$ value \\
\hline Regional and local & $5(83.3 \%)$ & $1(16.7 \%)$ & 0.022 \\
Systemic & $5(29.4 \%)$ & $12(70.6 \%)$ & \\
\hline
\end{tabular}

related deaths and recurrences compared to other studies $[8,11,15]$. Therefore, this study could not show the independent role of established prognostic factors except the nodal stage.

\section{Conclusion}

This study showed cytoplasmic expression of YB-1 was associated with more aggressive tumor and poor overall survival in breast cancer. Therefore, high cytoplasmic expression of YB-1 in cancer cell could be used as an independent prognostic marker for predicting OS in breast cancer. Furthermore, developing a new targeting agent for YB-1 in cancer cell could be a novel progress in breast cancer treatment.

Acknowledgments This study was supported by a grant of the Korean Health Technology R and D Project, Ministry of Health and Welfare, Republic of Korea (HI12C0050).

\section{Compliance with ethical standards}

Conflict of interest The authors declare that they have no conflict of interest.

Open Access This article is distributed under the terms of the Creative Commons Attribution 4.0 International License (http://crea tivecommons.org/licenses/by/4.0/), which permits unrestricted use, distribution, and reproduction in any medium, provided you give appropriate credit to the original author(s) and the source, provide a link to the Creative Commons license, and indicate if changes were made.

\section{References}

1. Merlo DF, Ceppi M, Filiberti R, Bocchini V, Znaor A, Gamulin $\mathrm{M}$, et al. Breast cancer incidence trends in European women aged 20-39 years at diagnosis. Breast Cancer Res Treat. 2012;134: $363-70$. 
2. Di CS, Baselga J. Management of breast cancer with targeted agents: importance of heterogenicity. Nat Rev Clin Oncol. 2010;7:139-47.

3. Eliseeva IA, Kim ER, Guryanov SG, Ovchinnikov LP, Lyabin DN. Y-box-binding protein 1 (YB-1) and its functions. Biochem (Mosc). 2011;76:1402-33.

4. Didier DK, Schiffenbauer J, Woulfe SL, Zacheis M, Schwartz BD. Characterization of the cDNA encoding a protein binding to the major histocompatibility complex class II Y box. Proc Natl Acad Sci USA. 1988;85:7322-6.

5. Kohno K, Izumi $H$, Uchiumi $T$, Ashizuka $M$, Kuwano $M$. The pleiotropic functions of the Y-box-binding protein, YB-1. BioEssays. 2003;25:691-8.

6. Shibahara K, Sugio K, Osaki T, Uchiumi T, Maehara Y, Kohno $\mathrm{K}$, et al. Nuclear expression of the Y-box binding protein, YB-1, as a novel marker of disease progression in non-small cell lung cancer. Clin Cancer Res. 2001;7:3151-5.

7. Kamura T, Yahata H, Amada S, Ogawa S, Sonoda T, Kobayashi $\mathrm{H}$, et al. Is nuclear expression of $\mathrm{Y}$ box-binding protein-1 a new prognostic factor in ovarian serous adenocarcinoma? Cancer (Phila.). 1999;85:2450-4.

8. Xie W, Yang J, Cao Y, Peng C, Ning H, Zhang F, et al. Expression of Y-Box-binding protein 1 in Chinese patients with breast cancer. Tumour Biol. 2012;33:63-71.

9. Oda Y, Ohishi Y, Saito T, Hinoshita E, Uchiumi T, Kinukawa N, et al. Nuclear expression of Y-box-binding protein-1 correlates with P-glycoprotein and topoisomerase II alpha expression, and with poor prognosis in synovial sarcoma. J Pathol. 2003;199:251-8.

10. Bargou RC, Jurchott K, Wagener C, Bergmann S, Metzner S, Bommert $\mathrm{K}$, et al. Nuclear localization and increased levels of transcription factor YB-1 in primary human breast cancers are associated with intrinsic MDR1 gene expression. Nat Med. 1997;3:447-50.

11. Dahl E, En-Nia A, Wiesmann F, Krings R, Djudjaj S, Breuer E, et al. Nuclear detection of Y-box protein-1 (YB-1) closely associates with progesterone receptor negativity and is a strong adverse survival factor in human breast cancer. BMC Cancer. 2009;9:410.

12. Li Y, Wen ZS, Yang HX, Luo RZ, Zhang Y, Zhang MF, et al. High expression of Y-box-binding protein-1 is associated with poor survival in resectable esophageal squamous cell carcinoma. Ann Surg Oncol. 2011;18:3370-6.

13. Yasen M, Kajino K, Kano S, Tobita H, Yamamoto J, Uchiumi T, et al. The up-regulation of Y-box binding proteins (DNA binding protein A and Y-box binding protein-1) as prognostic markers of hepatocellular carcinoma. Clin Cancer Res. 2005;11:7354-61.

14. Tay WL, Yip GW, Tan PH, Matsumoto K, Yeo R, Ng TP, et al. Y-Box-binding protein-1 is a promising predictive marker of radioresistance and chemoradioresistance in nasopharyngeal cancer. Mod Pathol. 2009;22:282-90.

15. Fujii T, Kawahara A, Basaki Y, Hattori S, Nakashima K, Nakano $\mathrm{K}$, et al. Expression of HER2 and estrogen receptor alpha depends upon nuclear localization of Y-box binding protein-1 in human breast cancers. Cancer Res. 2008;68:1504-12.

16. Wu J, Lee C, Yokom D, Jiang H, Cheang MC, Yorida E, et al. Disruption of the Y-box binding protein-1 results in suppression of the epidermal growth factor receptor and HER-2. Cancer Res. 2006;66:4872-9.

17. Lee C, Dhillon J, Wang MY, Gao Y, Hu K, Park E, et al. Targeting YB-1 in HER-2 overexpressing breast cancer cells induces apoptosis via the mTOR/STAT3 pathway and suppresses tumor growth in mice. Cancer Res. 2008;68:8661-6.

18. Ito T, Kamijo S, Izumi H, Kohno K, Amano J, Ito K. Alteration of Y-box binding protein-1 expression modifies the response to endocrine therapy in estrogen receptor-positive breast cancer. Breast Cancer Res Treat. 2012;133:145-59.

19. Yu YN, Yip GW, Tan PH, Thike AA, Matsumoto K, Tsujimoto $\mathrm{M}$, et al. Y-box binding protein 1 is up-regulated in proliferative breast cancer and its inhibition deregulates the cell cycle. Int $\mathrm{J}$ Oncol. 2010;37:483-92.

20. Gu C, Oyama T, Osaki T, Kohno K, Yasumoto K. Expression of Y-box-binding protein-1 correlates with DNA topoisomerase II alpha and proliferating cell nuclear antigen expression in lung cancer. Anticancer Res. 2001;21:2357-62.

21. Kosnopfel C, Sinnberg T, Schittek B. Y-box binding protein 1-a prognostic marker and target in tumour therapy. Eur J Cell Biol. 2014;93:61-70.

22. Gopal SK, Greening DW, Mathias RA, Ji H, Rai A, Chen M, et al. YBX1/YB-1 induces partial EMT and tumourigenicity through secretion of angiogenic factors into the extracellular microenvironment. Oncotarget. 2015;6:13718-30.

23. Khan MI, Adhami VM, Lall RK, Sechi M, Joshi DC, Haidar OM, et al. YB-1 expression promotes epithelial-to-mesenchymal transition in prostate cancer that is inhibited by a small molecule fisetin. Oncotarget. 2014;5:2462-74.

24. Evdokimova V, Tognon C, Ng T, Ruzanov P, Melnyk N, Fink D, et al. Translational activation of snail1 and other developmentally regulated transcription factors by YB-1 promotes an epithelialmesenchymal transition. Cancer Cell. 2009;15:402-15.

25. Yi SY, Ahn JS, Uhm JE, Lim do H, Ji SH, Jun HJ, et al. Favorable response to doxorubicin combination chemotherapy does not yield good clinical outcome in patients with metastatic breast cancer with triple-negative phenotype. BMC Cancer. 2010;5(10):527. 